\title{
FATIGUE CRACK GROWTH ASSESSMENT BY THE FEA-BASED SIMULATION OF DAMAGE ACCUMULATION
}

\author{
Sergei Petinov ${ }^{1,2}$, Ruslan Guchinsky ${ }^{2}$, Igor Korolev ${ }^{2}$ \\ ${ }^{1}$ Department of Strength of Materials, Institute of Civil Engineering, St.Petersburg Polytechnic University,

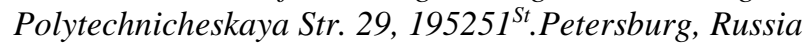 \\ ${ }^{2}$ Numerical Simulation of Deformation and Failure of Materials and Structures Lab, \\ Institute for Problems in Mechanical Engineering, Russian Ac. of Sci. \\ Bolshoy Pr.61, 199178 St.Petersburg, Russia \\ E-mail:" spetinov@mail.ru (corresponding author)
}

\begin{abstract}
Fatigue crack extensions are typically predicted by application of the Linear fracture mechanics techniques. However, there is a problem of numerical estimations of the crack three-dimensional shape and front extension, which becomes insoluble when the crack approaches the back face of the analyzed detail. Also, considering material plasticity, especially at the stage preceding the through thickness crack extension or complete fracture of a detail, is beyond the scope of the technique.

An approach based on the FEA simulation of fatigue damage accumulation has been developed. It allows assessing the crack initiation and propagation until complete failure of the detail affected. The approach is illustrated considering the example of fatigue failure of the non-continuous fillet-welded joint with incomplete penetration of weld material. The crack initiation in the cavity, its three-dimensional shape formation and evolution are simulated taking into account the elastic-plastic cyclic deformation of weld material until almost complete failure of the joint. The results of the analysis are in good agreement with the published experimental data.
\end{abstract}

Keywords: FEA-based simulation of fatigue; strain-life criterion for fatigue; fatigue damage accumulation technique; fatigue crack growth; fatigue of welded joints; fillet-welded joints.

\section{Introduction}

In the current codes for fatigue assessment of welded structures in different applications (in civil engineering, in ship and marine technologies, etc.) a set of S-N curves characterizing fatigue strength of parent material and of typified welded joints is applied, e.g., (DNV, 2010), etc. These «design» S-N curves are obtained by fatigue testing of specimens until almost complete failure in two pieces. Thus, the criteria ( $\mathrm{S}-\mathrm{N}$ curves) comprise the number of load cycles prior the crack initiation and crack propagation. Respectively, when applied for fatigue analysis of structural details, the current approach does not provide evaluation of the extent of damage. E.g., in (DNV, 2010) a suggestion is presented: «For practical purpose one defines the failures in test data as being crack growth through the thickness. When this failure criterion is transferred into a crack size in a real structure where some redistribution of stress is more likely, this means that this failure criterion corresponds to a crack size that is somewhat less than the plate thickness».

A more specific problem in fatigue analysis of structures emerges when load-carrying fillet-welded joints are subjected to extensive alternating loading, particularly, the joints with incomplete penetration of weld material. In such joints fatigue cracks frequently initiate at the slit, weld root, and propagate through the weld unobserved over a certain period. Estimation of fatigue life of structural components, where fillet-welded joints are located in the areas of stress concentration, is complicated by uncertainty in the slit geometry and in defining the stress controlling the crack initiation. Of special importance are the non-continuous part- penetration joints where the stress flow passes via the weld material.

These features of analysis and the damage development are regarded highly menacing for structural integrity, although in the manufacturing procedures filletwelded joints are considered «friendly for production» and are frequently used in many cases.

In attempts to provide fatigue analysis of load-carrying fillet-welded joints, design codes, e.g., (DNV, 2010), etc. recommended application of the Linear fracture mechanics methodology. Considering continuous uniformly loaded joints, the stress analysis might be reduced to the generalized plane stress problem. The crack path can be approximately predicted by the FEA as passing along the maximum principal stress planes through the weld throat. Respectively, the stress intensity factor (SIF) values over the crack path should be calculated. Further, the crack extensions are found by integrating the Paris equation, (see DNV, 2010). It should be immediately noted that the crack (SIF) analysis in this case is limited by the properties of stress field at the crack tip: the stress singularity vanishes as the crack traverses approximately 0.7 of the weld throat; apart from that increasing stress in the ligament actuates material plasticity, thus limiting the applicability of the Linear fracture mechanics and the prospects of analysis.

Fatigue analysis is essentially more complicated when the crack initiation is expected at the weld root of the non-continuous and non-uniformly loaded joints. In such cases, planning fatigue and $\mathrm{FE}$ analysis of the joint, one has to foresee the crack initiation loci and the three-dimensional crack progress until it reaches the outer surface of the weld bead. The mesh should be re-arranged following the crack front evolution and extensions. 
Again, the stress field singularity range may be limited and material plasticity would prevent the feasibility of analysis well before the crack might have approached the outer surface of the weld bead.

A prospective approach to fatigue analysis of welded details where the crack initiation and propagation should be considered may be based on the development and application of the damage accumulation principle in conjunction with the finite element formal modeling of fatigue properties of the polycrystalline material structure.

\section{Development of the approach}

The prospects of fatigue crack growth simulation by the damage accumulation procedure were considered promising through the past decades, e.g. (Glinka, 1982), (Ellyin, 1997), etc. Implementation of the finite-element modeling (FEM) provided the necessary technique for simulation. However, the mentioned analyses considered crack extensions in a homogeneous material along the axis of symmetry in the stress field. The application of FEM for the analysis of crack propagation when the crack path might be affected by the inhomogeneous development of damage or by specifics of the stress field immediately assumed instantaneous rearrangement of the finite element grid during the crack extensions.

Meanwhile, applicability of such approach is limited by a class of problems where there is no correlation between the crack path and the previous history of damage accumulation in a material. When crack extensions depend on the history of damage accumulation, the readjustment of the $\mathrm{FE}$ grid is unacceptable since it leads to the loss of the information on the developing degradation of material in the volume ahead of the crack tip.

This work focuses on further development of the approach considering material cyclic plasticity, inhomogeneous plasticity in polycrystalline material, and the three-dimensional crack extensions, in welded joints in particular. Within the scope of the work, a version of specialized FE meshing was proposed for the application in plane stress which, on the one hand, would allow to naturally save the information on fatigue damage in material (finite elements) prior to failure and to model the whole process of fatigue crack development, and on the other hand, would minimize the influence of the grid on the fatigue crack traces.

Firstly, we tested the ideas of the approach in simulation of fatigue of polycrystalline silicon components of microstructures. Recently, in a series of investigations it was found that material (thin film structure components) can degrade and fail under cyclic loading. Observations indicated initiation and propagation of fatigue cracks in the oxide film developed in the stress concentration in the course of cyclic loading, e.g., (Muhlstein et al, 2001), etc. Experiments had shown the «sloped» character of test results, typical of metallic materials, increasing fatigue life of test samples with decreasing stress amplitude. Test results also revealed essential scatter of fatigue lives, covering several decimal orders. Other important observations were made (George, et al 2006), where cyclic plasticity of silicon was observed and studied.

These and other experimental findings allowed suggesting an approximate procedure of finite-element based simulation of fatigue of silicon components. To exemplify the procedure the one-side notched plate of the type tested in (Muhlstein et al, 2001) was modeled by a composition of quadrilateral elastic-plastic finite elements provided with random variable fatigue resistance properties. These had to be obtained from the results of testing of the notched samples.

As shown in (Muhlstein et al, 2001), fatigue lives of the tested specimens cover the range of $10^{5}-10^{11}$ of the load cycles while the average stress amplitude varies from 4.5 GPa to approximately $2.0-2.2 \mathrm{GPa}$. The latter was regarded the threshold value below which no failure occurred. The elastic-plastic cyclic properties of the material at the test conditions were unknown; we assumed bi-linear cyclic curve with proportionality stress range corresponding to the local stress range at the notch root calculated when the «nominal» stress amplitude was 2.2 GPa. The strain-hardening part of the cyclic curve slope was assumed one tenth of the elastic one; evolution of cyclic properties was not considered. The fatigue properties of the «material structure elements», FEs, were described by the Coffin-Tavernelli's equation (Petinov, 2003):

$\Delta \varepsilon=C N^{-\alpha}+S_{t h} / E$

where $\Delta \varepsilon$ is the strain range, $C$ and $\alpha$ are the material empirical «constants», to be obtained from the (Muhlstein et al, 2001) data, $S_{t h}$ is the mentioned threshold stress range.

Fatigue process in the implied «material elements» was modeled as the sequence of damage accumulation in FE's using the Palmgren-Miner rule:

$d=\sum_{i}\left(n_{i} / N_{i}\right)$

where $n_{i}\left(\Delta \varepsilon_{i}\right)$ is the number of load cycles with the strain range, $\Delta \varepsilon_{i}$, characteristic of an «i» element, accordingly, its location in the FE model, found from elastic-plastic analysis; $N_{i}\left(\Delta \varepsilon_{i}\right)$ is the number of cycles prior failure of «i $\rangle$ element, the latter obtained from (1). Consequently, the damage accumulated in every element:

$d=\sum_{i}\left(n_{i} / N_{i}\right)=C^{-1 / \alpha} \cdot \sum_{i} n_{i} \cdot\left(\Delta \varepsilon-S_{t h} / E\right)^{1 / \alpha}$

When the damage index in a sequential element attained $D=1$, the stiffness of the element decreased by several decimal orders; it indicated the crack extension, and after a series of element «failures» accelerated the progress of the damage and crack growth rate, it was regarded the specimen failure.

The scatter of test results was allowed by assuming the constant $\mathrm{C}$ in (1) a randomly selected value from the limited population for every of the FEs according to the experimental data. The parameters of the equation (1) 
were estimated based on the results (Muhlstein et al, 2001) and the mentioned procedure: $\alpha=0.085$, and mean value of the parameter $\mathrm{C}=0.6022$. The FE model of the specimen part at the notch is shown in Fig.1. Its enlarged fragment illustrates the damage and crack progress at the notch root.

The analysis of the damage accumulation and the «crack growth» rate provides an assumption that the accumulation of damage in elements occurs only at the first steps of the «crack» extensions. Further, the damage accumulation is concentrated completely in the element located at the crack tip and due to high value of exponent $(1 / \alpha=11.8)$ it provides high rate of the crack progress, which might be regarded a fracture.

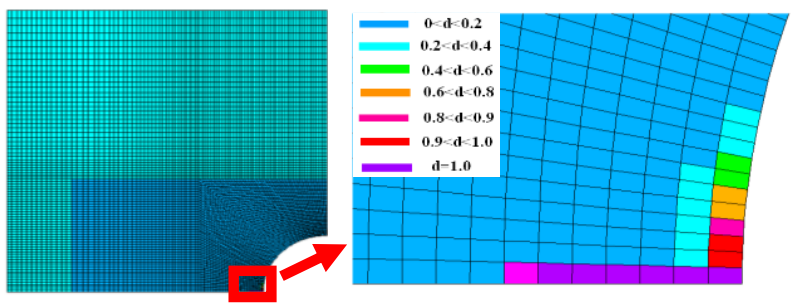

Fig. 1. FE model of specimen; damage intensity and the crack progress.

In order to simulate the test results scatter, the parameter $\mathrm{C}$ attributed to FEs, «material elements», was assumed evenly distributed in the range $(0.8 \ldots 1.2) \mathrm{C}$. The results of simulation are shown in Fig. 2, compared with the test results (Muhlstein et al, 2001).

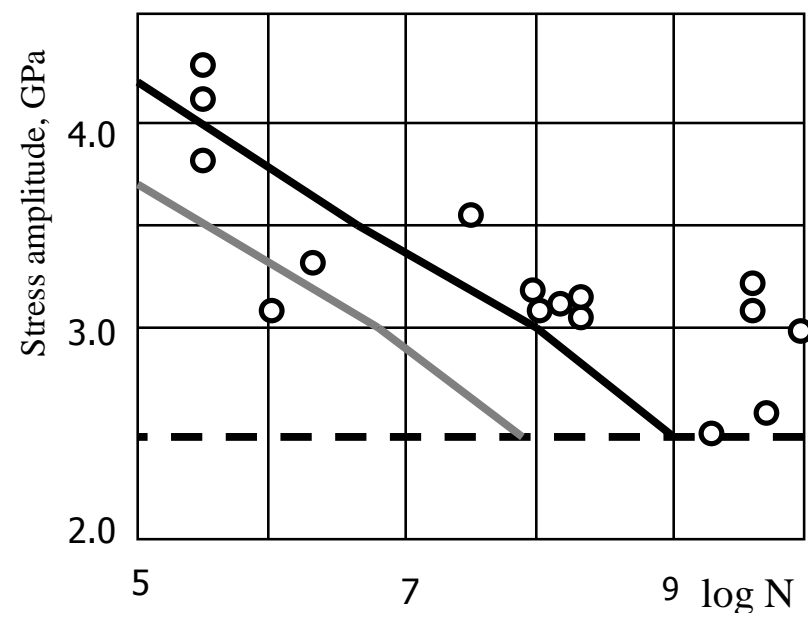

Fig. 2. Solid black line: constant $\mathrm{C}$ the same in elements; solid grey line - constant $\mathrm{C}$ randomized in elements, failure starts in elements with $\mathrm{C}=0.8 \mathrm{C}_{\text {mean }}$; circles - test results.

Comparison of results in Fig. 2 does not show a perfect correspondence; however, the approach may be regarded a promising one. What is notable, by varying the Coffin's constant $(\mathrm{C})$ it is possible to simulate the scatter of test results controlled by the properties of material element located at the stress concentration area. In our analysis, the difference in fatigue properties of elements at the notch itself is not the controlling process factor; the most important is the property of an element at the maximum stress concentration location. The threshold stress might be regarded a random value to allow for the scatter of fatigue strength, specifically seen in the range of fatigue lives, $\mathrm{N}=10^{9}-10^{11}$ cycles. Also, the cyclic elastic-plastic properties in the simulation procedure are assumed fairly provisional; experimental data are necessary.

In the above-presented analysis the crack initiation and growth were assumed to occur along the symmetry axis. A different scenario was realized in the case when a sharp notch was suggested at a certain distance from the axis of symmetry, Fig. 3. This turn an automatically generated regular finite element grid is used; as seen, the crack follows strictly the mesh nodal line, inclined to the direction of loading and direction of the maximum principal stress.

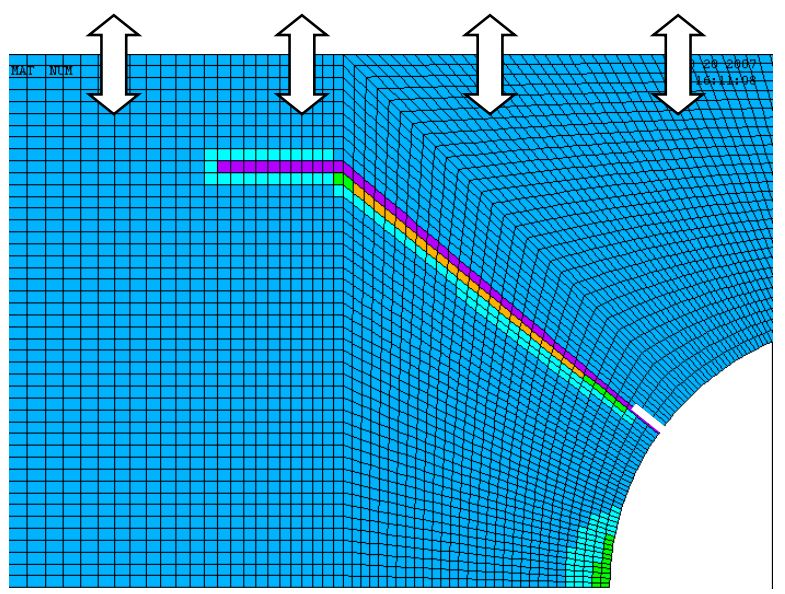

Fig. 3. Crack trace controlled by the mesh

A more detailed analysis (by using finer mesh at assumed crack tip) reveals that the most intensive damage accumulation at every crack increment develops in successive element located directly at the crack path extension, i.e. along the grid nodal line.

In order to minimize the influence of grid topology on the crack path, the following requirements for the meshing were formulated:

1. The crack tip should be provided «freedom» to turn under the influence of the local stress field and the rate of damage accumulation in the successive elements;

2. The isotropy of the grid should be provided; any specified nodal lines in the mesh would control the crack extensions.

Based on these requirements a cell structure of the mesh was derived as shown in Fig. 4 (on the left). The cell consists of «blocks-circles», in the center of each there are triangular elements which allow for deviation of the crack tip. By this in the center of every «block-circle» the crack path would be corrected according to the local stress field; further, the crack would extend towards the next «block-circle». 

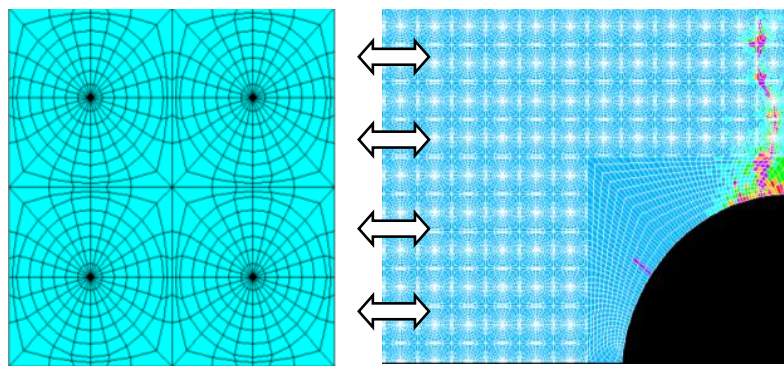

Fig. 4. Left: FE cell of the proposed «isotropic» mesh type; right hand: crack initiation and growth from the «smooth» stress concentration.

If the size of the grid cell is small enough, the crack trajectory slightly differs from the «true» one. Also this cell is consistent with the requirement to the mesh isotropy, since there is no predominant direction, nodal point line, capable of controlling the crack path. Fig. 4, on the right, illustrates crack growth history in the plate with a circular hole, where the isotropic grid was applied. In this example the initial «defect» (magenta line) occurs inactive, and the crack is initiated by the damage accumulation mechanism in the «proper» location. Further development of the «isotropic» FE mesh is given in (Korolev et al, 2008).

Next, the approach was applied for the numerical simulation of fatigue process in non-continuous nonuniformly loaded fillet-welded joint, where the crack was assumed to be initiated in cavity, at the weld root. For reference, configuration and results of fatigue testing of welded specimens reported in (Petinov, et al, 2006) were used for numerical simulation of fatigue. The damage accumulation was based on the elastic-plastic cyclic properties of the material; the crack shape and extensions were simulated until almost complete failure of the joint, when the crack approached the outer surface of the weld and propagated along the weld in the throat plane.

Fatigue resistance of material, a low-carbon structural steel electrode material, is characterized by the Strain-life Manson's criterion (Petinov, 2003):

$\Delta \varepsilon=\Delta \varepsilon_{p}+\Delta \varepsilon_{e}=C N^{-\alpha}+B N^{-\beta}$

Experimentally obtained parameters of which are: $C=0.480, B=0.011, \alpha=0.653, \beta=0.140$ (Petinov,

2003); $\sigma_{y}=290, M P a, \sigma_{u}=450, M P a, \Delta \varepsilon$ is the total cyclic strain range, $\Delta \varepsilon_{e}, \Delta \varepsilon_{p}$ are the elastic and plastic strain range components, respectively; $N$ is the number of cycles prior to fatigue failure of material, defined by the pre-established decrease of the specimen stiffness.

The specimen characteristics and loading mode given in (Petinov, et al, 2006), Fig. 5, were used to design the FE model of the joint. Attention was focused solely on the crack initiation at the slit tip circumference and propagation towards the outer surface of the weld bead. It was assumed that crack propagation surface insignificantly deviated from the weld throat plane.
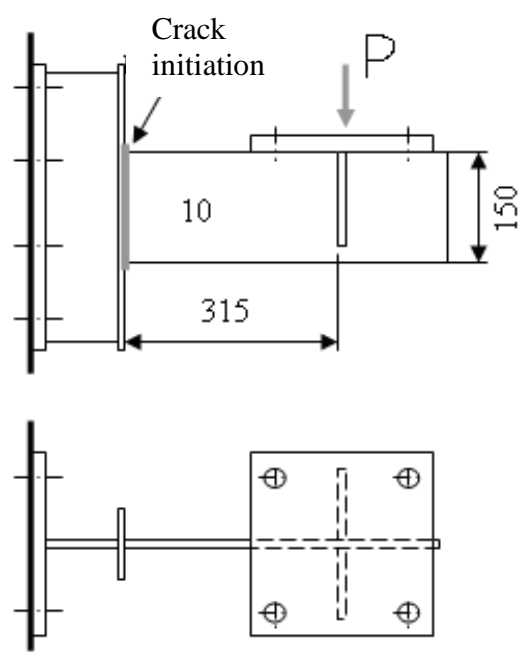

Fig. 5. Specimens tested and reported in (Petinov et al, 2006).

The fracture modes in (Petinov et al, 2006) were indicated only schematically; therefore the fractographic observations revealed in testing of similar fillet-welded joints where fatigue cracks propagated from the weld root (Fricke, et al, 2004) were used to support the above assumption, Fig. 6.

Respectively, the mesh was designed reasonably fine in the expected growth plane to provide the necessary cyclic stress and strain resolution; otherwise, it was coarsened, as shown in Fig. 7, to reduce the processing time.

In the first load application, the elastic-plastic behavior of elements of the model was characterized by the static tensile diagram. In unloading, the elements, which exhibited plasticity in the initial phase, were further provided with generalized cyclic curve (Petinov, 2003). Further on, the calculated strain range was used to obtain the initial fraction of damage in the affected «material elements».

The number of load cycles corresponding to crack initiation at the slit circumference is $N_{o}$; then the damage accumulated at this step in the remaining affected elements:

$d_{\text {initial }}=N_{0} / N_{i}$

So far, $n_{i}=N_{0}$ is the initial part of the "fatigue process», $N_{i}$ is the number of load cycles to failure in the successive strain range conditions, in every «material element» where plasticity occurred in the initial and sequential loadings, which develop due to «failure» of elements. As in the above presented examples, the «failure» is defined by the condition:

$$
d=d_{\text {initial }}+\sum_{i}\left(n_{i} / N_{i}\right)=1
$$

Here $n_{i}=n_{i}\left(\Delta \varepsilon_{i}\right)$ is the number of load cycles corresponding the strain range $\Delta \varepsilon_{i}$, which completes the 
damage accumulation in a particular FE («material element»), $d$, at every «crack extension».

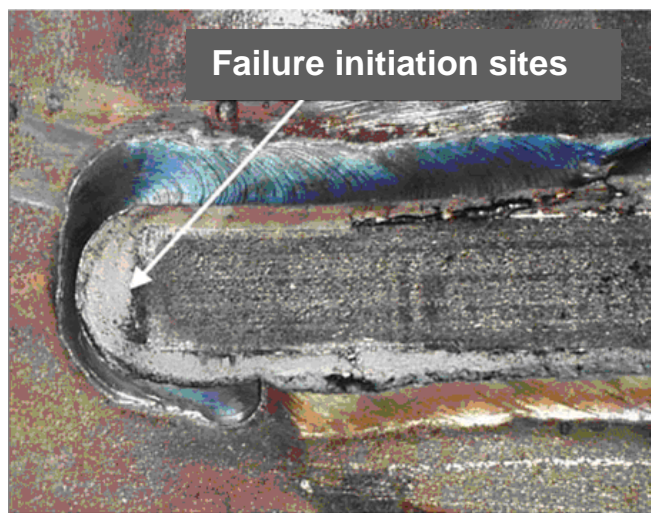

Fig. 6. Fracture of weld material initiated at the front of the cavity (Fricke et al, 2006)

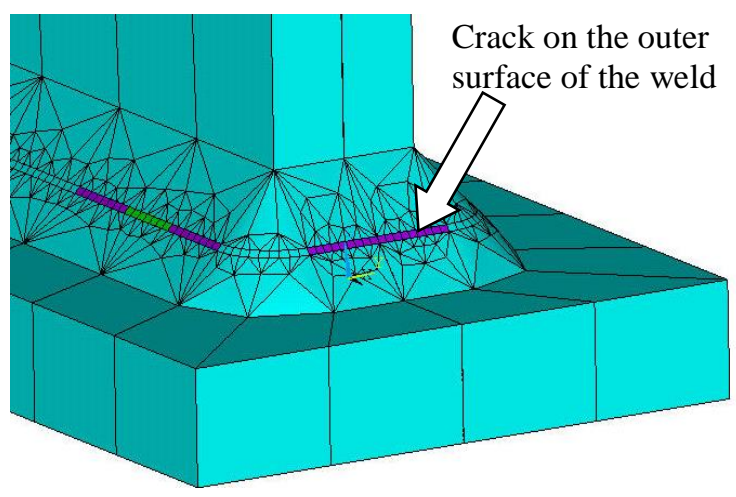

Fig. 7. FE model of the weld ending where crack initiated in the cavity caused by the lack of penetration of weld material.

\section{Results of simulation of the fatigue process.}

The briefly displayed procedure allowed analyzing the crack «initiation» at the cavity circumference and its extensions until almost complete fracture of the joint in the weld throat surface. Fig.8 shows the results of FE simulation of the crack initiated in several locations of the slit circumference which propagated towards the outer surface of the weld. As seen, the crack initiation is predicted not only at the weld ending inner boundary; also, simultaneous crack start is expected in a short distance from the weld ending, at the lateral tips of the cavity. This corresponds to the experimental findings (Fricke et al, 2006) shown in Fig. 6.

The crack shape evolution shown in Fig. 8 corresponds to $32,57,85,94,98$ and $99 \%$ of the total fatigue life of the joint. Same were the general crack shape characteristics, although the crack extension steps depended on the load range.

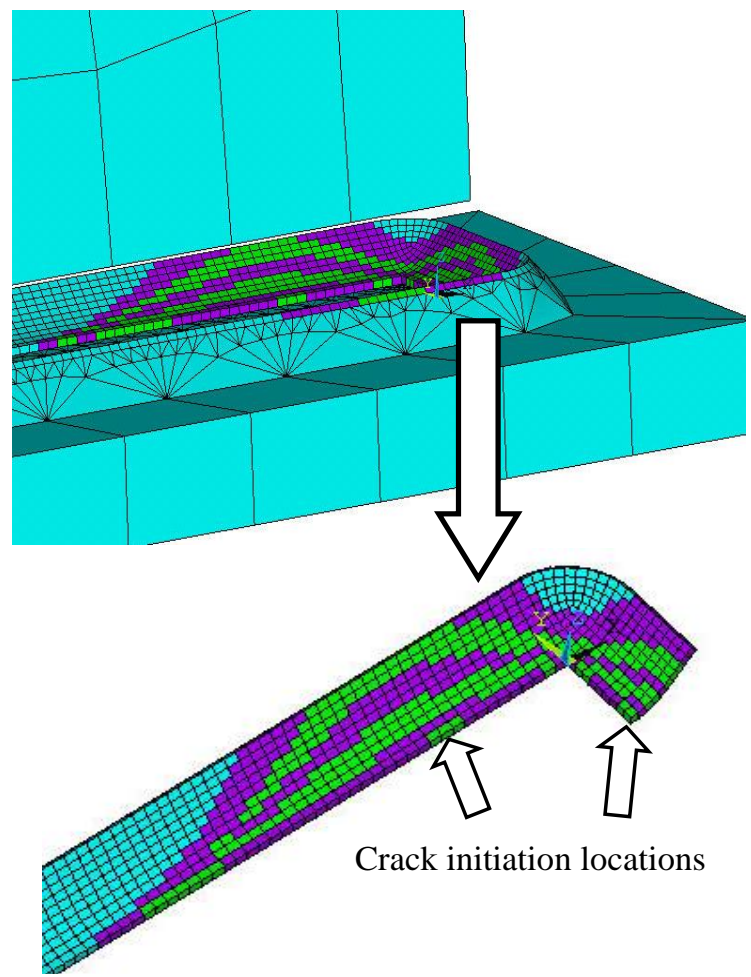

Fig. 8. Sequence of «failure» of elements and of the respective «crack extensions».

The results of numerical simulation together with the experimental data are presented in Fig.9. It is seen that the fatigue modeling by the damage accumulation in «material elements» fits reasonably well the experimental data.

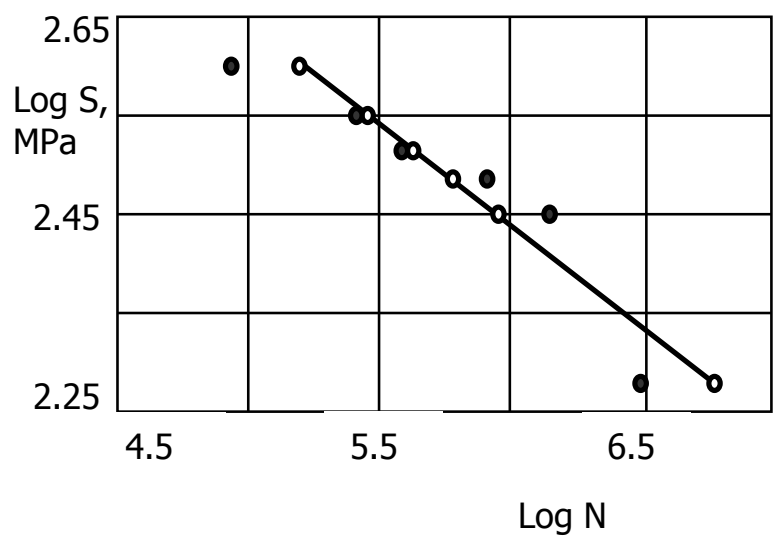

Fig. 9. Fatigue lives: bold circles, the test data (Petinov et al, 2006); solid line - results of the present numerical modeling 


\section{Conclusion}

The developed procedure provides reasonable approximations in fatigue analyses particularly important when the material plasticity should be considered in crack initiation and propagation. The approach is free from limitations unavoidable when the crack growth is analyzed by applying the Linear fracture mechanics techniques. Of special importance may be regarded the practical problem of fatigue assessment of load-carrying fillet-welded joints with incomplete penetration, which may be solved by numerical simulation of the fatigue process. Similarly, further development of the approach may provide clarifying the mentioned above uncertainties in the definition of the crack size corresponding termination of the design or residual fatigue life of structural components.

Concluding, it should be noted that repeating of the testing of a series of specimens at the same load conditions, as known, would result in a scatter of test data. The sources of scatter in case of welded joint may be attributed mostly to the difference of the volume and the shape of weld material, especially at the weld ending, to the difference of particular size and shape of cavity in every specimen tested. Those were uncertain; for the purposes of a more detailed analysis the geometry of the weld and slit should be assessed, e.g., by means of ultrasonic scanning.

Also, the present analysis did not consider residual welding stress and its influence on the fatigue process. However, it may be a factor of secondary importance in modeling fatigue of incomplete penetration welding joints: it was shown (Karzov et al, 1993) that the most influential component of welding stress, normal to the expected crack propagation plane, is insignificant at the cavity tip, increasing towards the outer surface of the weld bead. Material cyclic plasticity induced at the crack front and in ligament would result further in the course of crack extensions in the residual stress re-distribution and relaxation, so that total effect of welding stress may be neglected.

\section{Acknowledgements}

The work was supported by the Russian Grant for Fundamental Research 12-08-00943a

\section{References}

Det Norske Veritas. 2010. Fatigue assessment of ship structures. Classification Notes Nr.30.7. 2010. Hovik, Norway.

Ellyin, F., 1997. Fatigue damage, crack growth and life prediction. Chapman \& Hall, London. 489 p.

Fricke, W.; Doerk, O.; Gruenitz, L., 2004. Fatigue strength investigation and assessment of fillet-welds around stiffener and bracket toes. In Proc. of Special FPSO Conference of OMAE. 2004, Houston, TX.

George, A.; Jacques, A.; Legros, M., 2006. Low-cycle fatigue in silicon: comparison with FCC metals. Fatigue and Fracture of Engineering Materials and Structures, 30, pp. 41-56. http://dx.doi.org/10.1111/j.1460-2695.2006.01075.x

Glinka, G., 1982. A cumulative model of fatigue crack growth. International Journal of Fatigue, April, pp. 59-67. http://dx.doi.org/10.1016/0142-1123(82)90061-5

Karzov, G.P.; Margolin, B.Z.; Shvetsova, V.A., 1993. Physical and mechanical modeling of the failure processes. Polytechnic Publ., St.Petersburg, 324 p. (in Russian)

Korolev, I.K.; Petinov, S.V.; Freidin, A.B., 2008. FEM simulation of fatigue damage in a polycrystalline silicon structure. In Proc., VI Intern. Conference on Reliability of Materials and Structures «RELMAS-2008». 2008, SPb Polytechnic University, St.Petersburg.

Muhlstein, C.L.; Brown, S.B.; Ritchie, R.O., 2001. High-cycle fatigue and durability of polycrystalline silicon thin films in ambient air. Sensors and Actuators, A 94. Elsevier, pp. 177-188.

Petinov, S. V., 2003. Fatigue analysis of ship structures. Backbone Publishing Co., P.O. Box 562, Fair Lawn, NJ 07410. 2003. 262 p.

Petinov, S.V.; Kim, W.S.; Paik, Y.M., 2006. Assessment of Fatigue Strength of Weld Root in Ship Structure: An Approximate Procedure. Ship and Offshore Structures Journal, Woodhead Publishing, 1(1), pp. 55-60. 